\title{
BOND DEGRADATION OF CORRODED REINFORCEMENT: AN EXPERIMENTAL AND NUMERICAL STUDY
}

\author{
Mohammed Abdellatef*, Elsayed Salem ${ }^{\dagger}$, David Lau*, Lauren Stenroos* AND Mohammed \\ Alnaggar* \\ ${ }^{*}$ Rensselaer Polytechnic Institute \\ Troy, NY USA \\ e-mail: alnagm2@rpi.edu \\ ${ }^{\dagger}$ Menoufiya University \\ Shebin El Kom, Egypt. \\ Visiting Scholar at Rensselaer Polytechnic Institute, Troy, NY USA \\ e-mail: saleme@rpi.edu
}

Key words: Corrosion, Cracking, Bond-Slip, Lattice Discrete Particle Model

\begin{abstract}
Corrosion of rebars in concrete is a major source of deterioration in structures exposed to extreme environmental conditions. It is one of the main life time limit states for many infrastructures. In addition to the well understood effect of section capacity reduction due to reduction of steel cross section, the loss of bond between rebars and concrete enables larger deformations that are not easily understood nor expected. To understand the problem, a series of accelerated corrosion experiments were conducted. Different concrete cylinder sizes were cast with \#3 rebars placed in the middle of the cylinders with specific bonded and unbonded lengths. The specimens were cured in water for 14 days at $55^{\circ} \mathrm{C}$. After curing, specimens were immersed in $2 \% \mathrm{NaCl}$ solution and electric current was passed through the rebars recording both voltage and current passing. Rebars were coated and only exposed over the contact area to guarantee a uniform corrosion profile along the concrete-rebar interface. Different corrosion levels were achieved by imposing electric current for different intervals. Following the accelerated corrosion process, rebar pull out tests were conducted in order to quantify the change in bond strength due to rebar corrosion. The free end displacement was measured against pull out force and bond-slip curves were developed. The corroded area was at the end of the rebar not in the middle, thus, the measured force represented only bond-slip behavior of corroded bar with no non-corroded rebar length passing through as opposed to most tests in literature. Finally, to simulate the bond-slip behavior, a corrosion dependent Bond-Slip model was formulated and implemented within the Lattice Discrete Particle Model framework. The model was able to represent the functional form of the response and helped understanding different mechanisms. This paper presents the results of this on going research and raises different questions along with identifying the technical and knowledge gaps that yet to be tackled for the overall corrosion/bond behavior to be fully understood, modeled and predicted.
\end{abstract}

\section{INTRODUCTION}

Corrosion of steel rebars in concrete is a major source of deterioration in reinforced concrete structures [1]. It can affect the ductility of concrete structures and cause local and global failure in some cases. The cost of maintenance and repair of corrosion affected structures is usually high. For example, the annual cost of 
concrete bridge decks and substructures rehabilitation due to corrosion in the United States goes up to 200 and 100 million dollars respectively [2].

Corrosion takes many forms in civil engineering applications, such as: Galvanic, stress cracking, hydrogen embrittlement, intergranular, pitting, and crevice corrosion [3]. Although the first one is the most common and easy to predict, pitting and crevice corrosion are the ones that have more impact on steel reinforced structures. Pitting and crevice corrosion causes localized cracks, which are harder to predict and detect. These localized cracks could initiate an undesirable sudden fracture failure mechanism.

Corrosion is an electrochemical process, that could be driven or suppressed by the surrounding environment [3]. Typically, steel rebars embedded in concrete have several protective conditions against corrosion. First, they passivate creating a thin film, protecting them from being oxidized. Second, pore water solution has a high $\mathrm{pH}$, reducing the cathodic potential. Finally, thick cover around rebars reduces relative diffusivity of concrete to $1 / 300$ [4].

During service life, several factors initiate and accelerate corrosion, such as chloride penetration and carbonation [5]. Both factors cause reduction in concrete pore solution $\mathrm{pH}$ that directly results in increasing the corrosion potential. Additionally, chloride ions break the passive film, which allows pitting corrosion to start [6]. Also, cracking of concrete under loading conditions increases diffusivity of oxidation ions, such as hydroxide ions.

Once corrosion starts, rebars starts to lose both design capacity and bond strength due to reduction of cross section area [5]. Also as corrosion products have a bigger volume than the corroding layer, their expansion produces internal pressure inside concrete, which widens preexisting cracks and results in concrete spalling.

Modeling of steel corrosion process inside concrete is a complicated process, which raises a lot of challenges, for example: 1) The diffusion of oxidation ions inside concrete, 2) effect
DOI 10.21012/FC9.048

of concrete cracking, 3) probability of pitting corrosion at specific time and location, 4) mechanical properties of corrosion products, and 5) effect of corrosion on bond strength. Different models have been introduced in literature that consider some of these factors [7, 8], but yet, a comprehensive and predictive model is still lacking. In this paper, modeling of the effect of corrosion on bond strength will be presented with emphasis on the effect of corrosion products induced pressure on change in bond strength. The electro-chemo-mechanical aspects leading to the evolution of the pressure as well as the multi-diffusion processes that are involved are beyond the scope of this paper but are the over arching goal of the current research effort.

\section{Research Scope}

This paper represents an on going research work aimed at better understanding of the governing relations between corrosion of the rebar and progressive change in bond strength. The work is a combination of multi-step experimental evaluation driven by numerical modeling. The following sections will describe in detail what has been achieved so far. The overall plan was based on the following two steps:

(1) Experimental evaluation of Bond-Slip behavior for short bonded rebars at different corrosion levels up to full splitting of concrete samples due to corrosion products expansion. In this step, two different sizes of concrete cylinders were used.

(2) Development of a Bond-Slip model capable of representing the different stages of the behavior during pull out testing at different corrosion levels

While performing these two steps, the following questions were raised:

(1) What is the relation between corrosion level and the induced internal pressure before full cracking?

(2) What is the effect of corrosion products on cohesive and frictional parts of the 
bond law?, does it change the friction coefficient between concrete and the rebar?, does the effect comes merely from reducing the height of dents in the rebar? or it is both?

(3) How cracking initiates and propagates?, is it mainly a hydraulic fracturing process? or just after full cracking is generated, corrosion products can flow. This particular question is related to the chemistry, compressibility and viscosity of the corrosion product/concrete debris mix surrounding the corroded rebar surface.

The following two sections describe the experiments that were performed so far then the state of progress in modeling.

\section{Experimental Program}

\subsection{Description}

Two concrete sizes were used. The first was cylinders with $100 \mathrm{~mm}$ diameter (4") and the second was using $150 \mathrm{~mm}$ diameter (6"). For the first group only pull out tests and uniaxial compression tests were performed. The second group included pull out, uniaxial, three point bending and splitting tests. Mix design used was based on Bonded Concrete Ready Mix Company standard NYS DOT HP 5000 mix design and it is described in Table 1. To help accelerate corrosion, Sodium Chloride was added to the mixing water to achieve $2 \%$ by weight of Chloride per mixing water weight.

Table 1: Concrete Mix Design per $\mathrm{m}^{3}$

\begin{tabular}{ll} 
Component & Amount \\
\hline Silica cement content $[\mathrm{Kg}]$ & 317.8 \\
Fly ash content $[\mathrm{Kg}]$ & 79.45 \\
W/C [-] & 0.5 \\
Fine aggregate content $[\mathrm{Kg}]$ & 674.04 \\
Coarse aggregate content $[\mathrm{Kg}]$ & 1011.06 \\
Air entraining agent $[\mathrm{ml}]$ & 706.29 \\
Retardant $[\mathrm{ml}]$ & 1044.25 \\
$\mathrm{NaCl}[\mathrm{Kg}]$ & 5.24 \\
\hline \hline
\end{tabular}

\subsection{Material characterization results}

Tests were performed according to the ASTM standards C39 and C496 for unconfined compression and splitting tension tests respectively. For the notched three point bending test, as shown in fig. 1 1 , two beams $150 \times 150 \times 530$ $\mathrm{mm}$ were cast and tested at 54 days of age. The test span was $450 \mathrm{~mm}$, the notch was $75 \mathrm{~mm}$ deep and $4 \mathrm{~mm}$ wide. The notch crack mouth opening displacement (CMOD) was monitored and used for test control beyond an initial load control of $10 \%$ of the expected peak load.

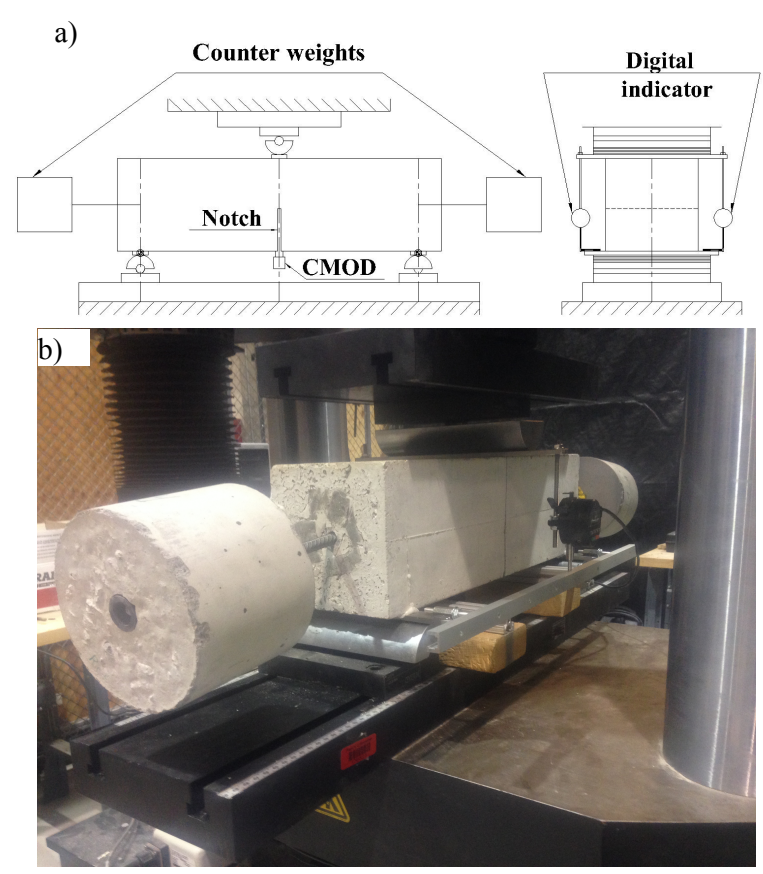

Figure 1: Three point bending test: a) Schematic drawing, b) actual specimen installed

To record the relative displacement between loading point and both supports, simply supported Aluminum beams were attached from both sides of the specimen to its lower support plates and to digital indicators were attached to the top loading plate with their plungers in full contact with the lower beams as shown in fig. 1 b. Two counter weights were attached at both ends of the specimen which allowed the recording of a very stable post peak tail down to less than $1 \%$ of the peak recorded load. Splitting and uniaxial compression tests were performed at 14 and 54 days of age while 3 point bend- 
ing test was only performed at 54 days. The characterized concrete average properties were $\left(f_{c}^{\prime}=32.37 \mathrm{MPa}, f_{s p}=3.47 \mathrm{MPa}\right.$ and $E_{c}=$ $29188 \mathrm{MPa})$ at 14 days, and $\left(f_{c}^{\prime}=24.38 \mathrm{MPa}\right.$, $f_{s p}=3.90 \mathrm{MPa}, E_{c}=29647 \mathrm{MPa}, G_{F}=153$ $\mathrm{N} / \mathrm{m}$ using the work of fracture method) at 54 days.

\subsection{Accelerated corrosion}

In this work, the corrosion level was defined as the reduction in rebar diameter due to the oxidation of iron outer layer. By integrating the electrical current passing through each rebar over time, the mass of oxidized iron is calculated using Faraday's law following [9], then the thickness of an equivalent external cylinder with height equal to the rebar bonded length is computed. The rebar diameter reduction ratio is then computed. For group I, only one corrosion level was achieved which was $3 \%$, while the next intended level was $6 \%$, the cylinders totally broke in splitting around $4.5 \%$ (identified from jump in current. For group II, four corrosion levels were produced $(2 \%, 4 \%, 10 \%$ and $46 \%)$. The last case caused splitting in concrete at about $10.5 \%$.

For each sample, a $330 \mathrm{~mm}$ (13") long \#3 rebar with $9.525 \mathrm{~mm}$ (3/8") diameter is used. The whole unbonded length of the rebar is coated using an anti-corrosion coating spray. The bonded length was $28.575 \mathrm{~mm}$ ( $3 \mathrm{~d}=9 / 8$ "). to prevent cone failure at the loaded end, a plastic pipe with a washer were used to cover 50 $\mathrm{mm}$ starting from the end of the bonded area. the space between the tube and the rebar is then filled with molten Paraffin wax. Next, the other end was exposed, rounded and threaded for loading in the testing frame. Finally, the rebar is inserted in the concrete plastic mold. Concrete is cast with a full height of $150 \mathrm{~mm}$ which guarantees more than $70 \mathrm{~mm}$ of top cover to the rebar end. This insures a nearly uniform corrosion of the rebar surface during the accelerated corrosion test. The steps of preparing the rebar are illustrated in fig. 2.

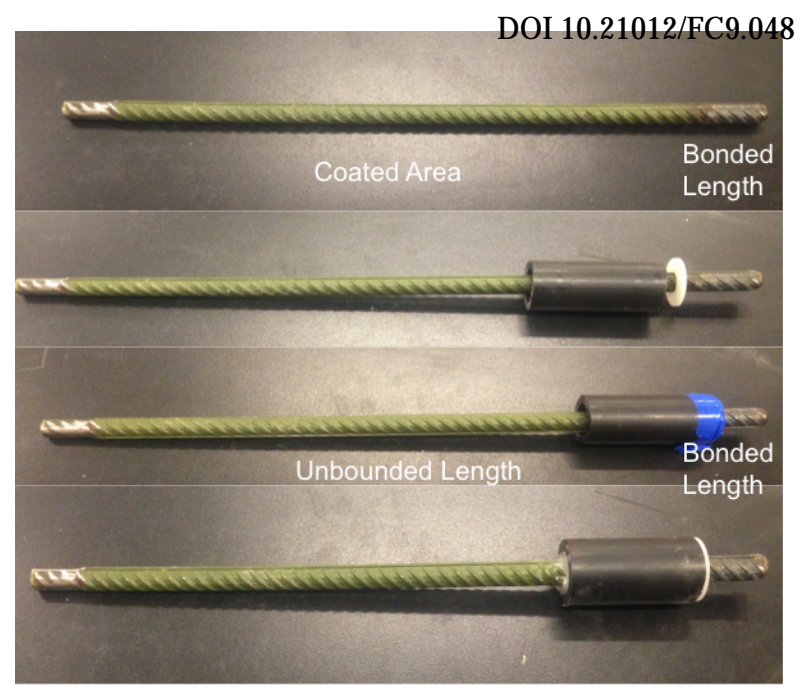

Figure 2: Steel rebar preparation

After 1 day of casting, the samples are demolded and then cured in water for 14 days at $55^{\circ} \mathrm{C}$. After curing, specimens were immersed in $2 \% \mathrm{NaCl}$ solution and electric current was passed through the rebars recording both voltage and current passing to determine the levels of corrosion expected. The reference specimens were kept in the same conditions but without any current passing.

\subsection{Pull out testing}

To measure the free end displacement, each specimen is prepared by cutting the additional concrete from the free end side. Carefully cutting about $10 \mathrm{~mm}$ away from the rebar end, then exposing the rebar end using drilling. The specimen is put upside down with the rebar passing through a $40 \mathrm{~mm}$ steel plate down through the moving head center hole. Inside the hole, a flange was fixed tightly to the rebar just below the plate and an LVDT was attached to the steel plate with its plunger touching the flange to record the relative displacement of the rebar loaded end and use it for test control. The threaded end of rebar was connected to a 25.4 $\mathrm{mm}$ (1") high strength threaded rod using a 50 mm coupling nut. The rod was then attached to the bottom fixed cross head using another 40 $\mathrm{mm}$ steel plate. The threaded rod was preloaded with a small load by turning the lower nut attaching it to the lower steel plate. 

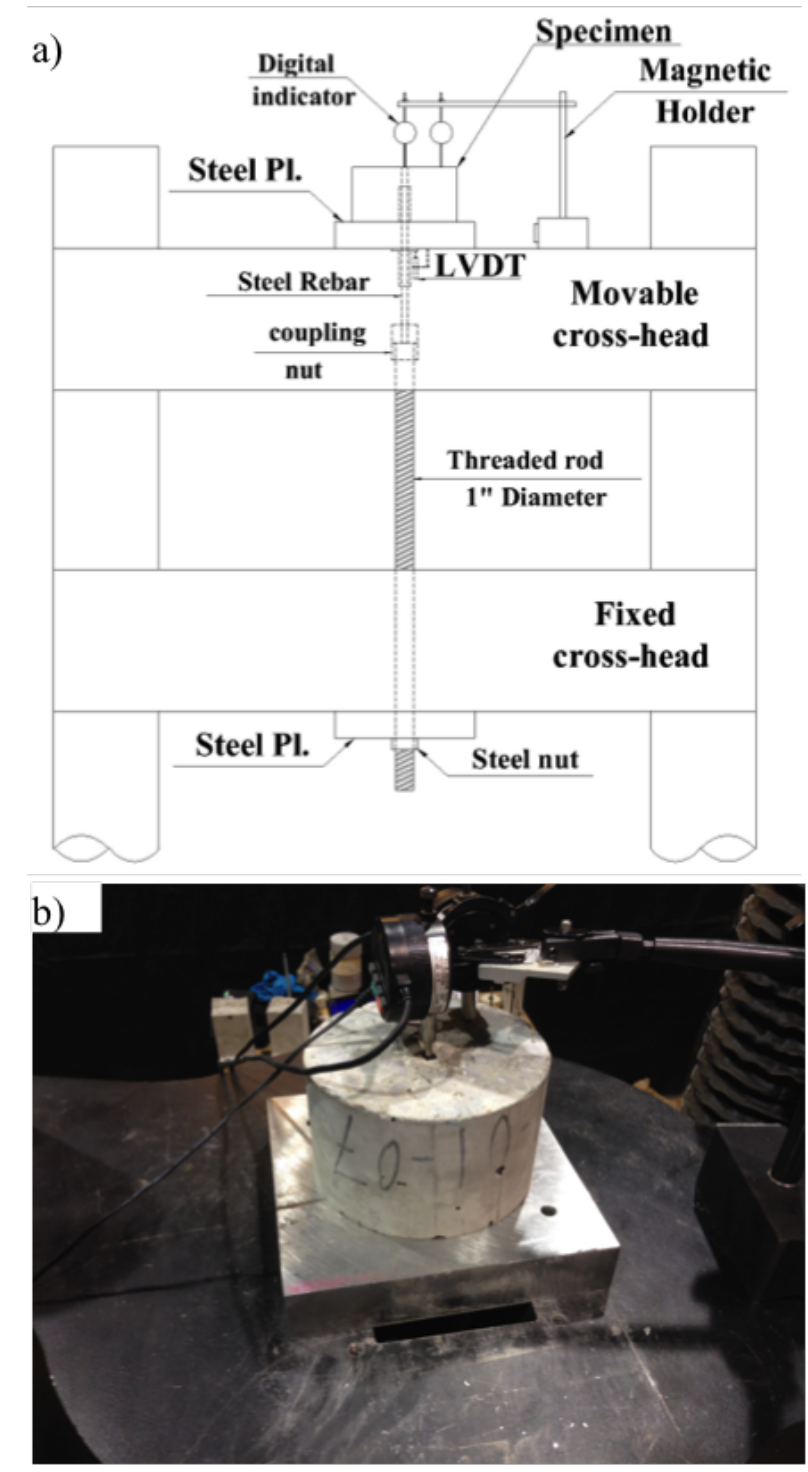

Figure 3: Pull-out test setup a) Schematic drawing, b) actual specimen installed with digital indicators at free end

After preloading, the test starts in load control up to $0.5 \mathrm{KN}$ then switched to displacement control using the LVDT reading. The free end displacement is recorded using two digital indicators held by a magnetic holder. One indicator measures the rebar end displacement and the other measures the concrete surface displacement and then the relative displacement is computed as the difference between these two readings. It has to be said that both the flange used to read the loaded end displacement and the setup for free end displacement were not accurate setups. The flange showed slight realignment during initial loading and the perpendicularity of
DOI 10.21012/FC9.048 the indicators to the free end of the rebar was not very accurate. The result is large variations in the initial elastic part as it is very sensitive and almost vertical. The test setup along with a schematic drawing is shown in fig 3 .

\section{Mechanical modeling}

The mechanical modeling consists of three different components, a) concrete model, b) rebar model, and c) bond-slip model. The rebar was simply described as an elastic beam element and the two other models are described here.

\subsection{The Lattice Discrete Particle Model (LDPM)}

The Lattice Discrete Particle Model (LDPM) [10,11] is a meso-scale discrete model that simulates the mechanical interaction of coarse aggregate pieces embedded in a cementitious matrix (mortar). The concrete mesostructure is geometrically represented through a system of polyhedral cells (see fig. $4 \mathrm{a}$ ) interacting through triangular facets and a lattice system composed by the line segments connecting the particle centers. In LDPM, rigid body kinematics is used to describe the deformation of the lattice/particle system and the displacement jump, $\llbracket \mathbf{u}_{C} \rrbracket$, at the centroid of each facet is used to define measures of strain as $e_{N}=\mathbf{n}^{\mathrm{T}} \llbracket \mathbf{u}_{C} \rrbracket / \ell$, $e_{L}=\mathrm{l}^{\mathrm{T}} \llbracket \mathbf{u}_{C} \rrbracket / \ell$ and $e_{M}=\mathbf{m}^{\mathrm{T}} \llbracket \mathbf{u}_{C} \rrbracket / \ell$. Where $\ell=$ interparticle distance; and $\mathbf{n}, \mathbf{l}$, and $\mathbf{m}$, are unit vectors defining a local system of reference attached to each facet. Next, a vectorial constitutive law governing the behavior of the material is imposed at the centroid of each facet. In the elastic regime, the normal and shear stresses are proportional to the corresponding strains: $t_{N}=E_{N} e_{N}^{*}=E_{N}\left(e_{N}-e_{N}^{0}\right) ; t_{M}=E_{T} e_{M}^{*}=$ $E_{T}\left(e_{M}-e_{M}^{0}\right) ; t_{L}=E_{T} e_{L}^{*}=E_{T}\left(e_{L}-e_{L}^{0}\right)$, where $E_{N}=E_{0}, E_{T}=\alpha E_{0}, E_{0}=$ effective normal modulus, and $\alpha=$ shear-normal coupling parameter; and $e_{N}^{0}, e_{M}^{0}, e_{L}^{0}$ are mesoscale eigenstrains that might arise from a variety of phenomena such as, but not limited to, thermal expansion, shrinkage, corrosion, sulphate attack and ASR expansion. For constitutive re- 
lations of stresses and strains beyond the elastic limit, one can refer to [10]. LDPM has been used successfully to simulate concrete behavior under a large variety of loading conditions. Furthermore it was successfully used to simulate different aging and deterioration phenomena including: Alkali-Silica Reaction (ASR) deterioration [12] and nondestructive evaluation [13]; and creep and shrinkage concrete behavior [14] In addition, LDPM was successfully used in structural element scale analysis using multiscale methods [15, 16] and reinforced concrete flexural and shear behavior [17];

\subsection{Modeling rebar and its interaction with concrete}

In this research, the experimentally measured values of rebar pull out forces were below the yielding strength of the used rebars, yet, a simple elastic-perfectly plastic material model was used in simulating the rebar as a 1D beam element with both translational and rotational degrees of freedom. To connect them together, a zero-thickness interface element with vectorial relationships is first formulated. The constitutive laws of the element are then formulated.

\subsubsection{Deformation Measures}

LDPM is a discrete model as described in the previous section. This allows it to naturally simulate fragmentation and large damage. When, concrete is highly fragmented and big cracks develop, the linear deformation pattern within the original tetrahedral element is no longer meaningful. A more general description for the material point motion is to consider the cells resulting from the Voronoi tessellation (see fig. 4a). Each cell is associated to the discrete particle it contains and is limited by triangular facets over which concrete constitutive equations are imposed. In the initial unstressed configuration, all LDPM cells fill the entire volume $V$. Again, each concrete material point is either inside a cell or may be shared with other cells if it is on the external surface of the cell. The motion of a point $\mathbf{x}$ inside a cell can be expressed in terms of velocities as

DOI 10.21012/FC9.048

$$
\mathbf{v}(\mathbf{x}, t)=\mathbf{v}_{P}(t)+\boldsymbol{\omega}_{P}(t) \times\left(\mathbf{x}-\mathbf{x}_{P}\right)
$$

where $\mathbf{x}_{P}$ is the position of the discrete particle at the center of the cell, $\mathbf{v}_{P}(t)$ and $\boldsymbol{\omega}_{P}(t)$ are its velocity and rotation rate vectors. This condition implies that all points inside a cell move rigidly with the cell. While this description may not account for the small deformations that occur in the elasto-plastic range it does a better job in describing the formation of concrete fragments in the post-failure regime and tracking their subsequent motion.

The motion of all material points inside rebars can be described as functions of the velocity and rotation rates of the points along the centerline. The velocity history on an arbitrary point $\mathbf{x}_{r}$ inside the rebar can be expressed as

$$
\mathbf{v}\left(\mathbf{x}_{r}, t\right)=\mathbf{v}\left(\mathbf{x}_{R}, t\right)+\boldsymbol{\omega}\left(\mathbf{x}_{R}, t\right) \times\left(\mathbf{x}_{r}-\mathbf{x}_{R}\right)
$$

where $\mathbf{x}_{R}$ is the projection of $\mathbf{x}_{r}$ on the axis of the rebar, and $\mathbf{v}\left(\mathbf{x}_{R}, t\right)$ and $\boldsymbol{\omega}\left(\mathbf{x}_{R}, t\right)$ are the velocity and rotation rate histories of $x_{R}$. The displacement history is obtained by integrating the velocity in time

$$
\mathbf{u}\left(\mathbf{x}_{r}, t\right)=\int_{0}^{t} \mathbf{v}\left(\mathbf{x}_{r}, \tau\right) d \tau
$$

For each material point inside the rebar or on its interface, there is a corresponding material point inside the concrete. The relative displacement $\mathbf{u}$ and relative velocity $\mathbf{v}$ functions are defined as:

$$
\begin{aligned}
& \mathbf{u}(\mathbf{x}, t)=\mathbf{u}_{c}(\mathbf{x}, t)-\mathbf{u}_{r}(\mathbf{x}, t) \\
& \mathbf{v}(\mathbf{x}, t)=\mathbf{v}_{c}(\mathbf{x}, t)-\mathbf{v}_{r}(\mathbf{x}, t)
\end{aligned}
$$

where the subscript $c$ and $r$ denote concrete and rebar points. Figure $4 \mathrm{~b}$ shows a schematic representation of a rebar (in red) and the surrounding LDPM tetrahedron (a 2D representative triangle is shown) with the concrete point denoted by c (blue dot) and the rebar point denoted by $r$ (red dot) with both axial and radial springs representing the constraints. For simplicity, constraints are imposed along the axis of the rebar (neglecting the rebar diameter effect) as will be described next. 


\subsubsection{Line Constraint Formulation}

In its simplest form, we postulate that the internal elastic energy associated to the constraints along the rebar axis can be expressed as a bilinear positive definite symmetric operator $a(\mathbf{u}, \mathbf{u})$ defined as

$$
a(\mathbf{u}, \mathbf{u})=\frac{1}{2} \int_{S} K \mathbf{u} \cdot \mathbf{u} d s
$$

where $K$ is a stiffness parameter representative of the local compliance of concrete at the rebar interface. The total work performed by the interactive forces $\mathrm{g}$ (force per unit rebar length) is expressed by $b(\mathbf{u})=\int_{S} \mathbf{g} \cdot \mathbf{u} d s$

For equilibrium, $a(\mathbf{u}, \mathbf{u})+b(\mathbf{u})$ must be 0 . For an arbitrary virtual displacement function $\delta \mathbf{u}$, we obtain

$$
\begin{aligned}
\delta a+\delta b & =a(\delta \mathbf{u}, \mathbf{u})+a(\mathbf{u}, \delta \mathbf{u})+b(\delta \mathbf{u}) \\
& =\int_{S} K \mathbf{u} \cdot \delta \mathbf{u} d s+\int_{S} \mathbf{g} \cdot \delta \mathbf{u} d s \\
& =\int_{S}(K \mathbf{u}+\mathbf{g}) \cdot \delta \mathbf{u} d s
\end{aligned}
$$

Since the expression above must be valid for an arbitrary displacement function $\delta \mathbf{u}$, then we have $\mathrm{g}=-K \mathbf{u} \quad$ for each $\quad \mathrm{x} \in S$.

For computational purposes, this expression can be discretized by subdividing the rebar into finite segments and by placing control points at the midpoint of each segment. For a segment of length $\Delta s$ we then have $\mathbf{f}=K \Delta s \mathbf{u}(\mathbf{x})$

This formulation can be extended to treat non linear conditions using different constitutive equations. The next section describes the constitutive behavior formulation considering bond-slip relations including corrosion effects.

\subsubsection{Corroded Bond-Slip Model (CBSM)}

The Corroded Bond-Slip Model (CBSM) is a set of constitutive relations that correlate the relative slip between concrete and steel rebar to bond stresses, which is based on [18].

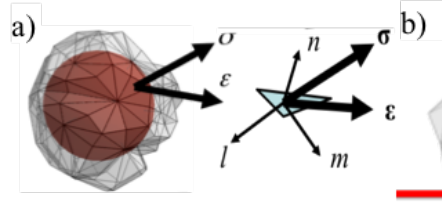

DOI 10.21012/FC9.048
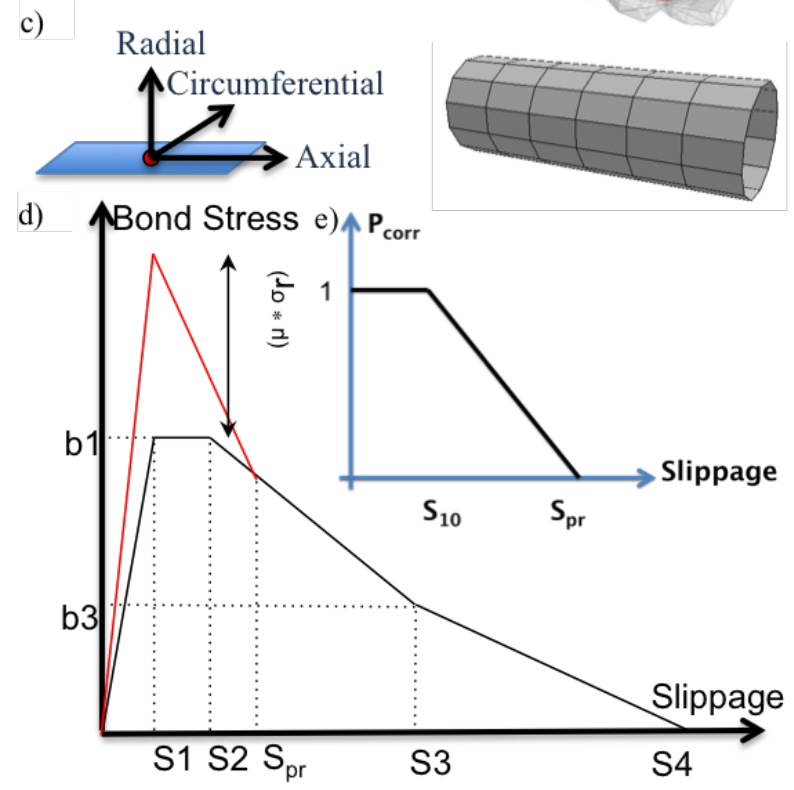

Figure 4: Mechanical models a) LPDM polyhedral cell, b) Schematic rebar-concrete interaction constraint, c) interface facets with slippage directions, d) Axial BondSlip constitutive behavior, and e) Pressure decay function

Following the previous definitions of relative deformation $\mathbf{u}$ and force per unit length $\mathbf{g}$, the surface of the rebar is subdivided into finite rectangular facets with areas $a_{f}$. The facets divide the rebar surface between two rebar nodes into axial and circumferential segments (see fig. 4c). For each facet, the stresses in vectorial form are given as $\sigma=\left\{\sigma_{a}, \sigma_{r}, \sigma_{c}\right\}$, where $\sigma_{a}$ is axial stress parallel to the rebar axis, $\sigma_{r}$ is the radial stress perpendicular to the rebar surface at the center of this facet, and $\sigma_{c}$ is circumferential stress tangential to the rebar surface at the center of this facet. The slippages in vectorial form are similarly given as $S=\left\{S_{a}, S_{r}, S_{c}\right\}$, where $S_{a}$ is axial slippage, $S_{r}$ is radial slippage, and $S_{c}$ is circumferential slippage. Simply, for a facet with a normal vector $\mathbf{n}_{\mathbf{f}}$ to its surface connected to a rebar segment parallel to a direction vector $\mathbf{n}_{\mathbf{r}}$, the slippages are related to $\mathbf{u}$ as $S_{a}=\mathbf{u} \cdot \mathbf{n}_{\mathbf{r}}$, $S_{r}=\mathbf{u} \cdot \mathbf{n}_{\mathbf{f}}$, and $S_{c}=\mathbf{u} \cdot\left(\mathbf{n}_{\mathbf{r}} \times \mathbf{n}_{\mathbf{f}}\right)$.

Similarly, the forces applied at the concrete 
nodes and their opposite ones along the rebar (that are summed over the perimeter and along the segment then lumped at both rebar segment ends) are computed as $\mathbf{f}=\sigma \cdot \mathbf{a}_{\mathbf{f}}$. Similar to the slippages, $\sigma_{a}$ is in $\mathbf{n}_{\mathbf{r}}$ direction, $\sigma_{r}$ is in $\mathbf{n}_{\mathbf{f}}$ direction and $\sigma_{c}$ is in $\left(\mathbf{n}_{\mathbf{r}} \times \mathbf{n}_{\mathbf{f}}\right)$.

The elastic behavior is given by:

$$
\left[\begin{array}{l}
\sigma_{a} \\
\sigma_{r} \\
\sigma_{c}
\end{array}\right]=\left[\begin{array}{ccc}
K_{a} & 0 & 0 \\
0 & K_{r} & 0 \\
0 & 0 & K_{c}
\end{array}\right]\left[\begin{array}{l}
S_{a} \\
S_{r} \\
S_{c}
\end{array}\right]+P_{\text {Corr }}\left[\begin{array}{l}
0 \\
p \\
0
\end{array}\right]
$$

Where $K_{a}$ is axial stiffness, $K_{r}$ is radial stiffness, $K_{c}$ is circumferential stiffness, and $p$ is the pressure produced from expansion of corrosion products.

The inelastic behavior is defined by individually defining constitutive relations for the different stresses and their interactions as follows:

To replicate the effect of concrete expansion due to rebar ribs an expansion coefficient $\mu_{N_{a}}$ as a function of the maximum absolute value of axial slippage $S_{a m}$ is used to evolve the radial stress as follows:

$$
\mu_{N_{a}}=\left\{\begin{array}{c}
\mu_{N}: S_{a m}<S_{20} \\
\mu_{N} \frac{S_{30}-S_{a m}}{S_{30}-S_{20}}: S_{20} \leq S_{a m}<S_{30} \\
0: S_{a m} \geq S_{30}
\end{array}\right.
$$

where $\mu_{N}$ is an expansion parameter, $S_{10}$ is the initial slippage before which the behavior is elastic, $S_{20}$ is the initial slippage at which bond starts to fail and transition into frictional behavior, $S_{30}$ is the axial slippage at which bond is lost and only frictional behavior is remaining, and $S_{40}$ is the slippage at which friction strength reaches it's minimum value of $b_{4}$ which is assumed to be zero (It is expected that the rebar segment is completely sheared off and larger debris have been crushed). Based on that, radial stress could be defined as $\sigma_{r}=K_{r}\left(S_{r}-\right.$ $\left.\mu_{N_{a}} S_{a m}\right)+P_{C o r r} p$, where $P_{C o r r}$ is a pressure release coefficient that is a function of slippage. It is assumed that within the elastic slippage part, $p$ is fully acting and after inelastic slippage starts $\left(S_{a m}>S_{10}\right)$, the pressure gradually releases over a shearing off slippage distance $\left(S_{p r}-S_{10}\right)$ (see fig. 4e). If the net radial stress is tension, and it's axial component is
DOI 10.21012/FC9.048 greater than axial bond strength, the bar would not be connected to concrete, and $\sigma_{a}$ is set to be 0 . Otherwise, axial stress will be dependent on level of confinement. Additionally, the friction force generated due to confinement will affect initial slippage limits. By adding the effect of radial stress then dividing by axial stiffness, updated slippages could be calculated as $S_{1}=S_{10}-\frac{\mu \times \sigma_{r}}{K_{a}}, S_{2}=S_{20}-\frac{\mu \times \sigma_{r}}{K_{a}}$, and $S_{3}=$ $S_{30}-\frac{\mu \times \sigma_{r}}{K_{a}}$ where $\mu$ is coefficient of friction between steel rebar and concrete. A schematic drawing of the axial stress constitutive behavior at zero radial stress (black line) and with the additional confinement due to $p$ (red line) is shown in fig. $4 \mathrm{~d}$. Similar to slippage, bond strength should be updated from it's initial values to account for confinement as $B_{1}=B_{10}-\left(\mu \sigma_{r}\right)$, and $B_{3}=B_{30}-\left(\mu \sigma_{r}\right)$, where $b_{10}$ is initial bond strength, $b_{1}$ is updated bond strength, $b_{30}$ is initial residual bond strength, and $b_{3}$ is updated residual bond strength. Bond stress is defined as a function of updated slippage and bond strength as following:

$$
\sigma_{b}=\left\{\begin{array}{cc}
b_{1}\left(1.0-\left(\frac{S_{1}-S_{a m}}{S_{1}}\right)^{\alpha}\right) & S_{a m}<S_{1} \\
b_{1} & S_{1} \leq S_{a m}<S_{2} \\
\frac{b_{1}\left(S_{3}-S_{a m}\right)+b_{3}\left(S_{a m}-S_{2}\right)}{S_{3}-S_{2}\left(S_{a m}-S_{3}\right)} & S_{2} \leq S_{a m}<S_{3}(9) \\
\frac{b_{3}\left(S_{4}-S_{a m}\right)+b_{4}\left(S_{a m}\right.}{S_{4}-S_{3}} & S_{3} \leq S_{a m}<S_{4} \\
b_{4} & S_{a m} \geq S_{4}
\end{array}\right.
$$

Finally, axial stress is computed for one of two cases: 1) if the redial component $\mu \sigma_{r}$ is less than $b_{30}$, so both $b_{1}$ and $b_{3}$ are positive, then $\left.\sigma_{a}=\min \left(\sigma_{b}, S_{a} K_{a}\right), 2\right)$ otherwise, if the redial component $\mu \sigma_{r}$ is greater than $b_{30}$ but less than $b_{10}$, so it is tension but still less than the bond strength, then $\sigma_{a}=\max \left(-\sigma_{b}, \min \left(\sigma_{b}, S_{a} K_{a}\right)\right)$.

The circumferential stress is still given as in the elastic case. So, with these definitions of stresses, forces at rebar nodes as well as concrete connected cells can be computed.

\section{Numerical Simulations}

Due to the limited amount of data from group I, it was only used for drawing general conclusions and qualitative comparison but no simulations were attempted to replicate it in this study. 


\subsection{Calibration of LDPM parameters}

LPDM parameters were calibrated only based on concrete testing at 14 and 54 days, then interpolated at the middle points at 28 and 35 days. Meso-scale elastic modulus $E_{0}$ was calculated assuming Poisson's ratio of 0.176, which gives $E_{0}=1.5455 E_{c}$. Assuming that fracture energy, $G_{f}=0.4 G_{F}$ [19], and $\sigma_{t}=f_{s p}$ [10], $l_{t}=2 E_{0} G_{t} / \sigma_{t}^{2}$. Finally, LDPM parameter $\sigma_{s} / \sigma_{t}$ was calibrated to achieve measured compressive strength $f_{c}^{\prime}[10,11]$. Other parameters were reasonably assumed based on [10]. Calibrated parameters are presented in table 2.

Table 2: Calibrated LDPM Parameters

\begin{tabular}{lcc}
\hline Parameter & 16 days & 54 days \\
\hline$E_{0}[\mathrm{MPa}]$ & 45109.75 & 45820.15 \\
$\sigma_{t}[\mathrm{MPa}]$ & 3.5 & 3.9 \\
$\sigma_{s} / \sigma_{t}[-]$ & 2.7 & 1.5 \\
$l_{t}[\mathrm{~mm}][-]$ & 436 & 356 \\
\hline \hline
\end{tabular}

\subsection{Calibration of CBSM parameters}

Calibration of CBSM parameters was performed in two stages. First stage is to calibrate initial bond-slippage parameters $\left(b_{10}, b_{30}\right.$, $b_{40}, S_{10}, S_{20}, S_{30}$, and $S_{40}$ ). Second stage is to calibrate internal confinement pressure corresponding to corrosion material expansion, as presented in table 3

Table 3: Calibrated CBSM Parameters

\begin{tabular}{lccccc}
\hline Age [Days] & 14 & 28 & 35 & 54 & 54 \\
Rebar $\Delta d$ & $(0 \%)$ & $(2 \%)$ & $(4 \%)$ & $(10 \%)$ & $(0 \%)$ \\
\hline$b_{10}[\mathrm{MPa}]$ & 14.9 & 11.31 & 10.86 & 9.56 & 13.5 \\
$b_{30}[\mathrm{MPa}]$ & 4.0 & 4.0 & 4.0 & 4.0 & 4.0 \\
$\alpha[-]$ & 2 & 2 & 2 & 2 & 2 \\
$S_{10}[\mathrm{~mm}]$ & 0.5 & 0.5 & 0.5 & 0.5 & 0.5 \\
$S_{20}[\mathrm{~mm}]$ & 0.9 & 0.9 & 0.9 & 0.9 & 0.9 \\
$S_{30}[\mathrm{~mm}]$ & 3.25 & 3.25 & 3.25 & 3.25 & 3.25 \\
$S_{40}[\mathrm{~mm}]$ & 12.0 & 12.0 & 12.0 & 12.0 & 12.0 \\
Pressure $[\mathrm{MPa}]$ & - & 63.0 & 68.5 & 40.0 & - \\
\hline \hline
\end{tabular}

In the first stage, the equations developed in the work of [20] have been used as start-
DOI 10.21012/FC9.048

ing point for calibration. In this work, a very similar bond-slip model was used and its parameters were experimentally correlated to concrete properties and rebar geometry. Rebar full cross section was considered to estimate the parameters before corrosion occurs. Following that, the reduction of rebar cross section was estimated assuming uniform corrosion over the bonded length, which allowed estimating the remaining rib thickness. The parameters $\left(b_{10}, b_{30}\right.$, $b_{40}, S_{10}, S_{20}$, and $S_{30}$ ) were then fine-tuned to match this research experimental results. Also, $S_{40}$ was introduced and calibrated to capture the bilinear behavior of the softening tail. Using the same procedure, CBSM parameter $b_{10}$ was scaled for different corrosion levels, while keeping the other parameters constant.
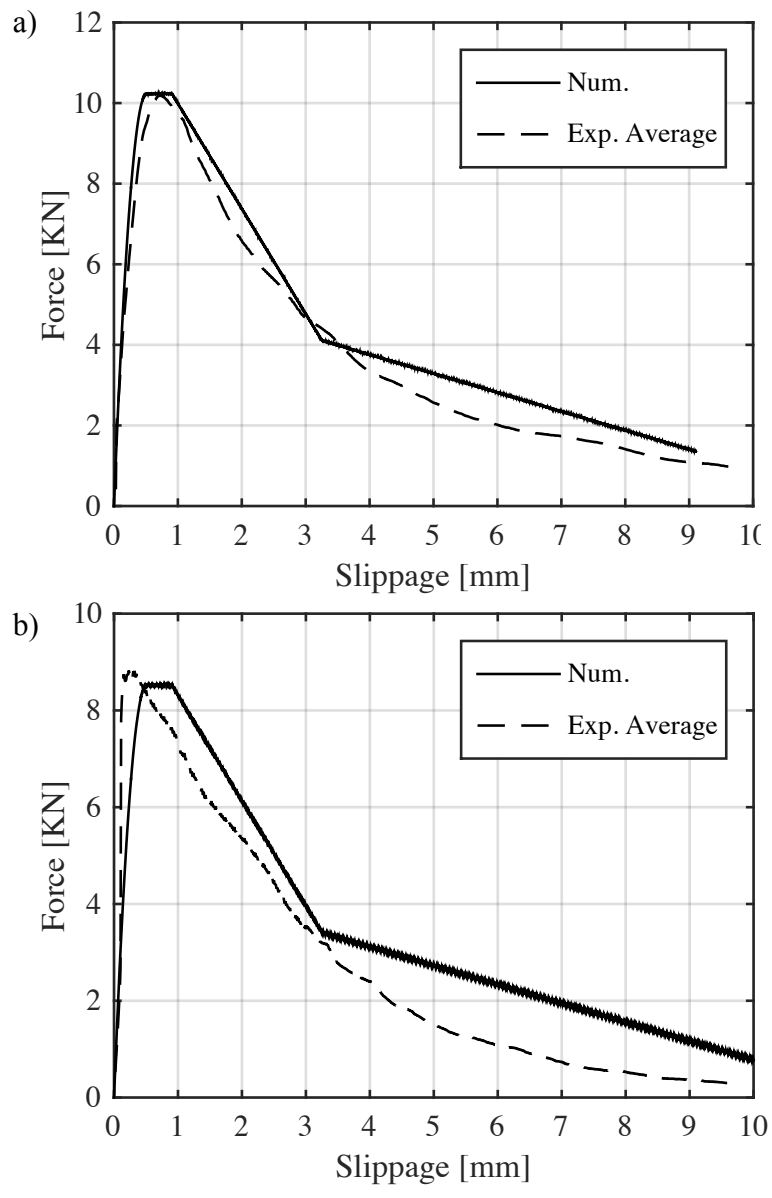

Figure 5: Experimental and Numerically simulated Force-Slippage responses for reference specimens (uncorroded) at a) 14 days, and b) 54 days 
This was based on the assumption that corrosion will impact directly the interlocking/cohesive behavior between concrete and steel rebar, but not the post shearing frictional behavior. The friction coefficient $\mu$ was assumed to be a constant typical value of 0.45 between concrete and steel. It was noticed that at initial corrosion levels, the effect of corrosion products pressure $p$ on bond increase is very high (abut two times the original value), so, to reduce the redundancy of the identification process, the expansion parameter $\mu_{N}$ was set to zero and its effect was assumed to be minimal compared to $p$ effect. The calibrated parameters are presented in Table 3 (columns 1 and 5). Experimental and numerical Force-Slippage curves are shown in fig. 5 which shows excellent agreement between experimental and numerical simulations.

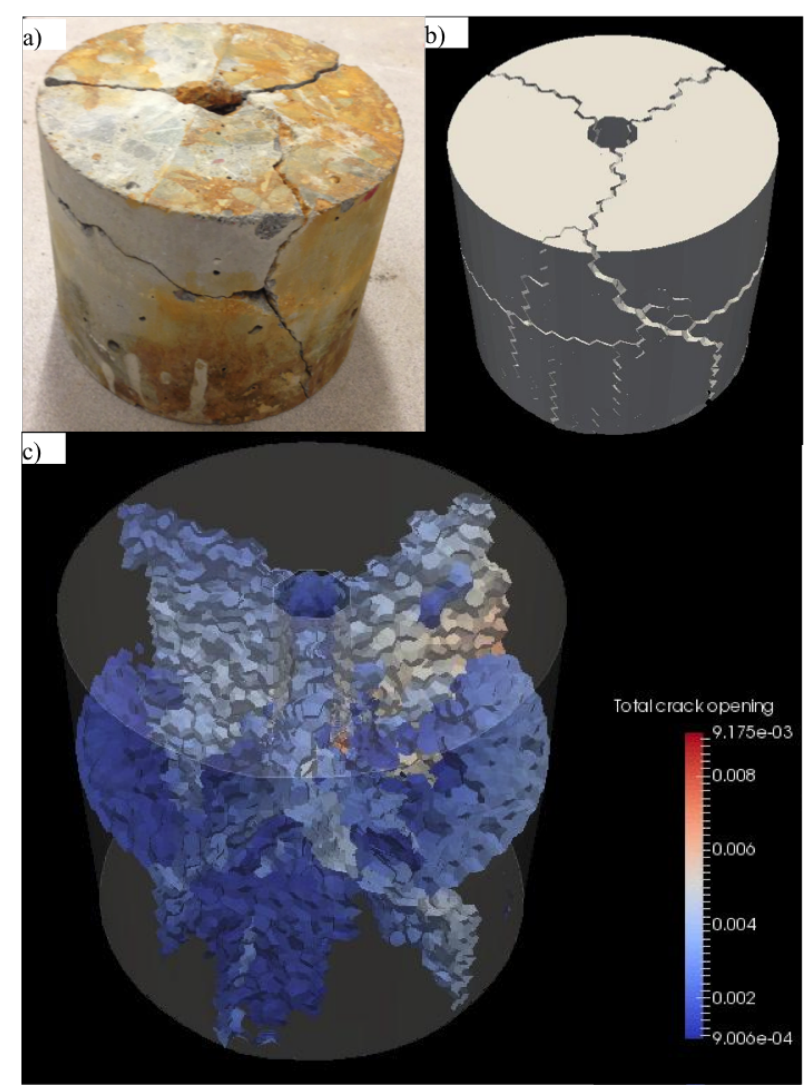

Figure 6: Splitting cracking due to corrosion: a) Actual specimen , b) Simulated surface cracking, and c) Internal crack openings $[\mathrm{m}]$

It has to be noticed that due to the initial
DOI 10.21012/FC9.048 realignment explained in the experimental program, the experimental elastic part is not always well captured. Nevertheless, the introduction of $S_{40}$ made it possible to perfectly capture the decay in the frictional part. Also, by comparing the peak bond forces, the 54 day peak is slightly less and that agrees with the reduced compressive strength reported. The main reason is the well known effect of using Chloride rich water in mixing and curing.

In the second stage, LPDM was used to estimate the upper bound value of the internal pressure that would cause the concrete cylinder to fully split. The pressure was applied at the interface between rebar and concrete to represent corrosion material expansion. This pressure represents a theoretical upper bound as it assumes no hydraulic fracturing before complete damage, nor corrosion products transport inside cracks. The simulated pressure at failure was199.5 MPa. The corresponding specimen that failed similarly in splitting, reached a corrosion level of $10.5 \%$. Figure 6 shows the actual specimen and its simulated crack pattern. At each corrosion level, the pressure parameter $p$ was calibrated by matching the experimental data. A good fit was obtained assuming $S_{p r}=S_{20}$. Calibrated parameters are reported in table 3 (columns 2,3 and 4). It was noticed experimentally that the bond peak increased from $10 \mathrm{KN}$ (no corrosion) to $24 \mathrm{KN}$ (at $2 \%$ ) then slightly increased to $25 \mathrm{KN}$ (at $4 \%$ ), then finally dropped to $17 \mathrm{KN}$ (at 10\%). This behavior is well explained by the final splitting of the sample at $10.5 \%$ of corrosion level. The reason is that at lower levels of corrosion, the concrete is still intact and is capable of holding the corrosion products within the vicinity of the rebar keeping the full pressure $p$ without relief. As corrosion progresses, the products start to either diffuse, hydraulically fracture concrete or both. The direct result is pressure relief. This was captured here in the model calibration as an increasing then decaying value of $p$ at different corrosion levels. Also, the identified values were all below the upper bound value of 199.5 $\mathrm{MPa}$ which indicates that the corrosion products 
are compressible and/or a diffusion/hydraulic cracking process is also slowly progressing.

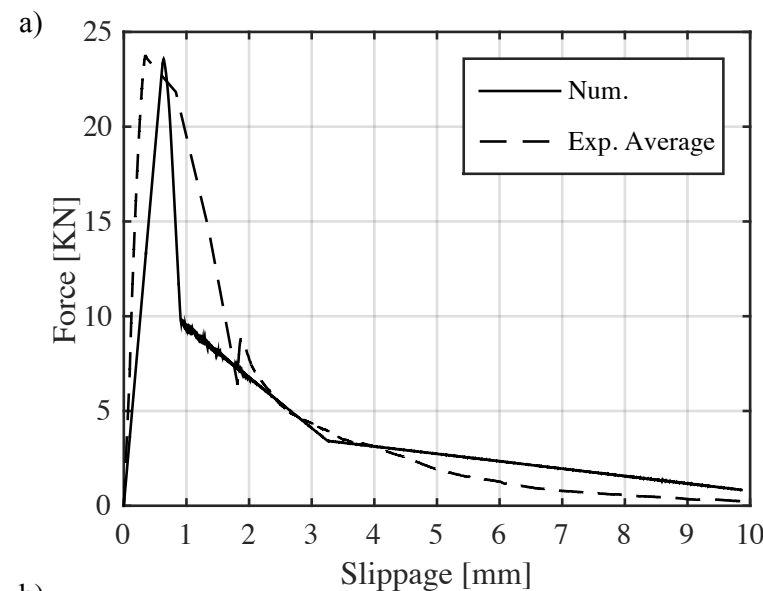

b)

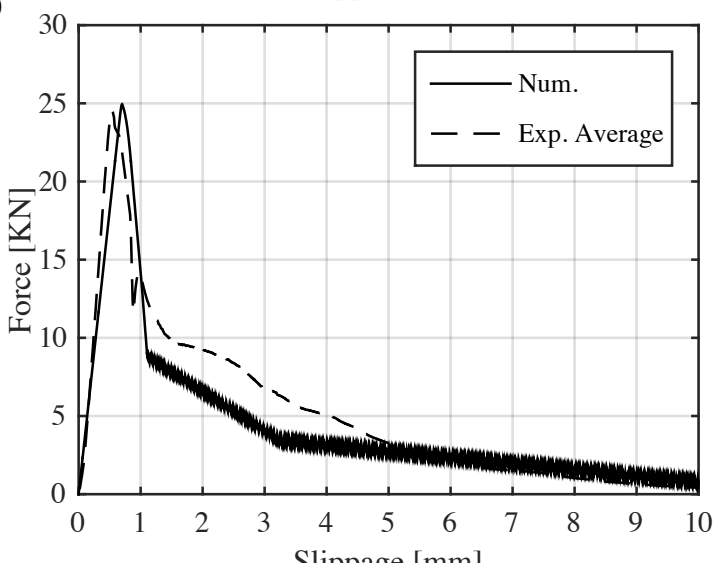

c)

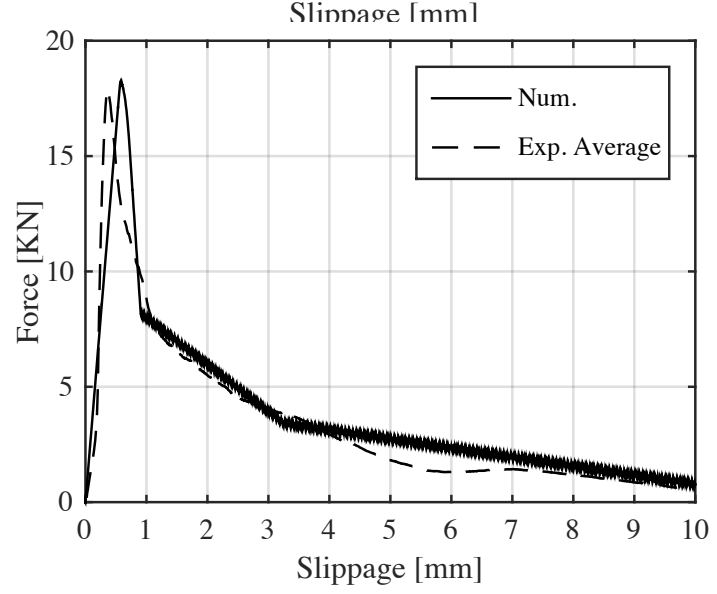

Figure 7: Experimental and Numerically simulated Force-Slippage responses for corroded specimens at a) $2 \%$, b) $4 \%$, and c) $10 \%$ corrosion levels

These questions are the subject of the ongoing study by the authors. But as an overall behavior, the model was able to capture the change in bond-slip behavior given the level of pressure as shown in fig. 7

DOI 10.21012/FC9.048

\section{Conclusion}

In this paper, an experimental evaluation of corrosion effect on rebar concrete bond strength was presented. Additionally, a bond-slip constitutive model was extended and implemented within the framework of the Lattice Discrete Particle Model (LDPM) to account for the effect of corrosion products expansion in the form of additional confining pressure. The model has been calibrated against the produced experimental data and it shoed well agreement with the functional form of the experimentally recorded bond-slip behavior. Additional experimental and numerical work is being pursued currently by the authors to evolve the pressure as a function of corrosion level and concrete strength considering the possibility of pressure relief due to hydraulic fracturing, diffusion of corrosion products and its compressibility.

\section{REFERENCES}

[1] Kyösti Tuutti. Corrosion of steel in concrete. Technical report, 1982.

[2] National Research Council (US). Committee on the Comparative Costs of Rock Salt and Calcium Magnesium Acetate (CMA) for Highway Deicing. Highway Deicing: Comparing Salt and Calcium Magnesium Acetate. Number 235. Transportation Research Board, 1991.

[3] Denny A Jones. Principles and prevention of corrosion. Macmillan, 1992.

[4] EJ Garboczi and DP Bentz. Modelling of the microstructure and transport properties of concrete. Construction and Building Materials, 10(5):293-300, 1996.

[5] Y Zhou, B Gencturk, K Willam, and A Attar. Carbonation-induced and chlorideinduced corrosion in reinforced concrete structures. Journal of Materials in Civil Engineering, 27(9):04014245, 2014. 
[6] CA Apostolopoulos and VG Papadakis. Consequences of steel corrosion on the ductility properties of reinforcement bar. Construction and Building Materials, 22(12):2316-2324, 2008.

[7] Zdenek P Bazant. Physical model for steel corrosion in concrete sea structurestheory. Journal of the Structural Division, 105(6):1137-1153, 1979.

[8] Joško Ožbolt, Filip Oršanić, and Gojko Balabanić. Modeling pull-out resistance of corroded reinforcement in concrete: Coupled three-dimensional finite element model. Cement and Concrete Composites, 46:41-55, 2014.

[9] J. B. Bushman. Calculation of corrosion rate from corrosion current (faraday's law). 2012.

[10] G. Cusatis, A. Mencarelli, D. Pelessone, and J.T. Baylot. Lattice discrete particle model (ldpm) for failure behavior of concrete. ii: Calibration and validation. Cement and Concrete Composites, 33(9):891-905, 2011.

[11] G. Cusatis, D. Pelessone, and A. Mencarelli. Lattice discrete particle model (ldpm) for concrete failure behavior of concrete. i: Theory. Cement and Concrete Composites, 33(9):881-890, 2011.

[12] Mohammed Alnaggar, Gianluca Cusatis, and Giovanni Di-Luzio. Lattice discrete particle modeling (ldpm) of alkali silica reaction (asr) deterioration of concrete structures. Cement and Concrete Composites, 41:45-59, 2013.

[13] Mohammed Alnaggar, Minghe Liu, Jianmin $\mathrm{Qu}$, and Gianluca Cusatis. Lattice discrete particle modeling of acoustic nonlinearity change in accelerated alkali silica reaction (asr) tests. Materials and Structures, pages 1-23, 2015.
DOI 10.21012/FC9.048

[14] M Abdellatef, M Alnaggar, G Boumakis, G Cusatis, G Di-Luzio, and R Wendner. Lattice discrete particle modeling for coupled concrete creep and shrinkage using solidification microprestress theory. 2015.

[15] Mohammed Alnaggar and Gianluca Cusatis. Automatic parameter identification of discrete mesoscale models with application to the coarse-grained simulation of reinforced concrete structures. 20th Analysis and Computation Specialty Conference, 36:406-417, 2012.

[16] Roozbeh Rezakhani and Gianluca Cusatis. Asymptotic expansion homogenization of discrete fine-scale models with rotational degrees of freedom for the simulation of quasi-brittle materials. Journal of the Mechanics and Physics of Solids, 88:320 345, 2016.

[17] Mohammed Alnaggar. Multiscale Modeling of Aging and Deterioration of Reinforced Concrete Structures. $\mathrm{PhD}$ thesis, NORTHWESTERN UNIVERSITY, 2014.

[18] Giovanni DiLuzio and Gianluca Cusatis. A new constitutive model for concretesteel bond behavior. Computational Modelling of Concrete Structures, Bicanic et al., Swets \& Zeitlinger, Lisse, 2003.

[19] Z.P. Bazant and E. Becq-Giraudon. Statistical prediction of fracture parameters of concrete and implications for choice of testing standard. Cement and Concrete Research, 32(4):529 - 556, 2002.

[20] S Lettow. Ein verbundelement für nichtlineare finite element analysenanwendung auf übergreifungsstöße (bond element for nonlinear finite element analysis-application to lap splices), university of stuttgart. PhD, Fakultät Bauingenieur und Umweltingenieurwissenschaften der Universität Stuttgart, Stuttgart, 2006. 